\title{
Food and feeding ecology of the neritic-slope forager black-browed albatross and its relationships with commercial fisheries in Kerguelen waters
}

\author{
Yves Cherel*, Henri Weimerskirch, Colette Trouvé \\ Centre d'Etudes Biologiques de Chizé, UPR 1934 du Centre National de la Recherche Scientifique, \\ 79360 Villiers-en-Bois, France
}

\begin{abstract}
Food and feeding ecology of black-browed albatrosses Diomedea melanophrys rearing chicks was studied during 2 austral summers (1994 and 1995) at the Kerguelen Islands. Dietary analysis and satellite tracking were used to estimate potential interactions with commercial fisheries in the area. Fish comprised $73 \%$ by fresh mass of albatross diet; other significant food items were penguins $(14 \%)$ and cephalopods $(10 \%)$. Twenty-one species of fish (232 individuals) were identified and included mainly nototheniid and channichthyid species. The most important were Dissostichus eleginoides (18.3\% by reconstituted mass), Channichthys rhinoceratus (16.9\%), Lepidonotothen squamifrons (11.6\%), and to a lesser extent, Bathyraja sp. (4.5\%) and Notothenia cyanobrancha (4.5\%). The cephalopod diet was dominated by 3 taxa, the ommastrephid squids Todarodes sp. (7.6\%) and Martialia hyadesi $(3.6 \%)$, and the octopus Benthoctopus thielei $(2.4 \%)$. Satellite tracking indicated that during trips lasting 2 to $3 \mathrm{~d}$, albatrosses foraged mainly over the outer shelf and inner shelf-break of the Kerguelen Archipelago. Birds moved to northern, eastern and southern waters, but never to the western Kerguelen shelf where there was a commercial longline fishery for $D$. eleginoides. Interactions with trawlers targetting $D$. eleginoides and Champsocephalus gunnari were of minor importance in the northern shelf. There, offal from $D$. eleginoides was available to the birds; fish and cephalopod bycatch were negligible. Most of the natural prey of black-browed albatrosses are primarily benthic and semipelagic organisms not known to occur near the surface. Since we demonstrate that most of them were not scavenged behind fishing vessels, the way albatrosses catch demersal organisms remains a mystery.
\end{abstract}

KEY WORDS: Cephalopods $\cdot$ Benthic prey $\cdot$ Diet $\cdot$ Fish $\cdot$ Procellariiformes $\cdot$ Satellite tracking

\section{INTRODUCTION}

Increasing exploitation of marine resources by man has focused attention on the management of marine ecosystems and the factors affecting them. Of major concern are the effects of competition between marine apex predators and fisheries, and the conservation of endangered species. In this context, accurate information on the diets and feeding ecologies of predators is essential if the effects of human activities are to be minimized. Fishery operations affect seabirds in 2 ways, negative and positive. The negative include inci-

*E-mail: cherel@cebc.cnrs.fr dental mortality (DeGange \& Day 1991, Robertson \& Gales 1998) and the competition for common resources (Montevecchi 1993); the positive include the provision of new resources through offal and discards (Blaber et al. 1995, Garthe et al. 1996).

Analysis of stomach contents of seabirds allows the determination of their main prey (Duffy \& Jackson 1986). Food can include some easily identified items associated with fisheries, such as fish and squids that are not naturally known from the area and are used as bait on longliners (Catard et al. 2000). More often, however, the identified species occur commonly in the foraging area and it is therefore a major challenge to separate the species naturally eaten from those made 
available by the fisheries. It is consequently generally assumed or hypothesized that prey known to be primarily benthic or semipelagic are items discarded during fishery operations (Croxall et al. 1995, Reid et al. 1996, Freeman 1998, Imber 1999), or adults that appear at the surface as a result of post-spawning mortality (Croxall et al. 1988), as already suggested for squids. The first assumption is rarely verified by working on the quantification of fishery waste and the corresponding importance of the discarded species in seabird diet at the same time (Thompson 1992). Moreover, information is lacking on the biology of some prey, thus inducing misinterpretation. It has been shown, for example, that small juvenile southern blue whiting Micromesistius australis, which were assumed to be taken by albatrosses in relation to a bottom trawl fishery at the Falklands (Thompson 1992), is more probably a natural prey there, as recently demonstrated in New Zealand waters (Cherel et al. 1999).

The diet of albatrosses is generally dominated by fish and cephalopods. Fish ranked first by mass in the diet of 3, and second in the diet of 6 albatross investigated so far (Cherel \& Klages 1998). Considering the importance of fish as prey, little qualitative and quantitative data are available on the contribution of different fish species to the diet of albatrosses. Generally, the stomach contents are digested, which precludes the identification of prey by the use of any external diagnostic features. Thus, either no, or very little fish remains have been identified to the species level in previous studies (Prince 1980, Thomas 1982, Harrison et al. 1983, Croxall et al. 1988, Hunter \& Klages 1989, Cooper et al. 1992, Ridoux 1994, Cooper \& Klages 1995, Reid et al. 1996, Green et al. 1998a, Imber 1999). Consequently, depending on the species considered, the quantitative composition of the fish diet of albatross is still poorly or essentially unknown (Reid et al. 1996).

The main objective of this study was to investigate the food and feeding ecology of the black-browed albatross Diomedea melanophrys, and to critically evaluate the importance of fishery waste and natural prey in its diet at the Kerguelen Islands, southern Indian Ocean. Black-browed albatrosses from the study colony at Canyon des Sourcils Noirs are known to feed mainly on fish (Weimerskirch et al. 1988), but no detailed information on the prey eaten is available in the literature. Traditionally, scientists working on piscivorous bird diets have tended to limit their research to the examination of otoliths only, disregarding skeletal remains. Various factors may affect the final qualitative and quantitative results of this method, leading to the underestimation, or ignorance of the presence of, some species and also to misleading results in back-calculation of fish sizes (Gosztonyi \&
Kuba 1998). We consequently made a reference collection of otoliths and bones for all the main species occurring in Kerguelen waters to help identify fishes. We also calculated allometric equations between otolith length (OL), mandible length (ML), standard length (SL) and fresh mass (M) to estimate the size of albatross prey. Interactions with fisheries were assessed by satellite tracking of adult albatrosses (Weimerskirch et al. 1997b), by comparison of the birds' foraging locations with fishery areas, and by quantifying birds attending vessels and fishery waste (Cherel et al. 1996, Weimerskirch et al. 2000).

\section{METHODS}

Study site, birds and field collection of samples. The study was conducted at the southern colony of Canyon des Sourcils Noirs $\left(49^{\circ} 41^{\prime} \mathrm{S}, 70^{\circ} 14^{\prime} \mathrm{E}\right)$, Jeanne d'Arc Peninsula, southern Kerguelen Islands, where 1000 to 1200 pairs of black-browed albatrosses breed annually (Weimerskirch et al. 1989).

Between 10 and 27 February 1994 and between 3 and 20 February 1995, 23 nests were observed continuously from dawn to dusk to monitor the visits of individual adults to their chick. One adult of each pair was marked on the breast with a patch of picric acid to make identification easier at a distance. The exact times of arrival and departure of the adults were noted continuously. All the chicks were weighed twice daily, in the early morning and late evening. Whenever feeding was observed, the chick was also weighed after the departure of the parents (see Weimerskirch et al. $1997 b$ for additional information).

A total of 114 dietary samples were taken from randomly-selected chicks after a returning parent had completed feeding them. Forty-five samples were collected in 1994 (37 in February and 8 in early April), and 69 samples in 1995 (47 in February and 22 in late March). Other prey items and additional incomplete stomach contents were also taken opportunistically during these periods. Samples were obtained by upending chicks over a plastic bucket and massaging the stomach and throat. All samples were returned deepfrozen $\left(-20^{\circ} \mathrm{C}\right)$ to the laboratory in France for analysis.

Dietary analysis. Each sample was thawed and drained, and accumulated cephalopod beaks were subsequently sorted. Beaks can persist in predator stomachs for weeks and even months, thus overemphasizing their importance in seabird diets. Following Cherel \& Klages (1998), accumulated beaks (without flesh attached) were consequently analysed separately from fresh items. These beaks were not considered when calculating the reconstituted proportion by mass in the diet of the different prey species. 
Fresh remains were divided into broad prey classes (fish, cephalopods, crustaceans, penguins and others), which were weighed to calculate their proportion by mass in the diet. Identification of prey relied almost totally on the examination of otoliths and bones for fish, keratinized beaks for cephalopods, and exoskeletons for crustaceans. Special care was made to use all fish hard parts recovered in stomach contents (bones, cartilageous elements, otoliths, scales, thorns, teeth), with an emphasis on some distinctive bones (premaxilla, maxilla, dentary, articular, parasphenoid, opercle, vertebrae and caudal skeleton) to identify items to the lowest possible taxon. As a result, 84.5\% ( $\mathrm{n}=196)$, $6.9 \%(\mathrm{n}=16)$ and $3.4 \%(\mathrm{n}=8)$ of the total number of fish prey $(\mathrm{n}=232$ ) were identified to the species, genus and family levels, respectively; only $5.2 \%(\mathrm{n}=12)$ of individuals remained undetermined.

In the same way, we used the morphology of both lower and upper beaks, instead of that of lower beaks only, to determine cephalopod prey. It was not possible to differentiate upper beaks or overly eroded lower beaks of juvenile Todarodes sp. from those of juvenile Martialia hyadesi. Accumulated beaks of Benthoctopus thielei could not be distinguished from those of the newly described species Graneledone gonzalezi (Guerra et al. 2000). However, all identifiable fresh remains of octopuses showed 2 rows of suckers on the arms (1 row in G. gonzalezi), thus indicating the occurrence of $B$. thielei only. We consequently assumed that all accumulated material was also beaks from $B$. thielei.

Items were identified by comparison with material held in our own collection and by reference to the available literature, including Andersen (1984), Iwami (1985) and Williams \& McEldowney (1990) for fish; Clarke (1986) for cephalopods; Clarke \& Holmes (1987), Baker et al. (1990), Branch et al. (1991), Vinogradov et al. (1996) and Murano (1999) for crustaceans. Length of uneroded or slightly eroded otoliths (OL) and dentary bones (ML for mandible length) of fish, lower rostral length (LRL) of squid beaks, lower hood length (LHL) of octopus beaks, and eye diameter or total length of crustaceans were measured with a vernier caliper or using an ocular scale in a binocular microscope. Fish standard length (SL), cephalopod dorsal mantle length (DML), crustacean total length and prey body masses (M) were calculated using regression equations (Clarke 1986, Adams \& Klages 1987, Rodhouse \& Yeatman 1990, Williams \& McEldowney 1990, Ridoux 1994, Jackson 1995, authors' unpubl. data). For the few species where no relationships were available, length and $M$ were estimated using equations for closely related species or for species with a similar morphology.

Satellite tracking. In February 1994 and 1995, 12 and 10 adult albatrosses, respectively, were fitted with satellite transmitters (8 T2038 Toyocoms and 2 Microwave 100s) weighing between 55 and $80 \mathrm{~g}$ (i.e. 1.5 to $2.2 \%$ of the birds' body mass). The transmitters were fitted on back feathers with adhesive tape and left on the birds for 1 to 5 successive foraging trips. Between 8 and 15 locations were received each day per transmitter, giving a total of 1290 and 857 locations in 1994 and 1995, respectively (for further information, see Weimerskirch et al. 1997b). Satellite fixes were obtained through the Argos System and the data were analysed following Weimerskirch et al. (1993).

Twenty-one dietary samples ( 7 and 14 samples in 1994 and 1995, respectively) were taken after foraging trips by adult birds fitted with satellite transmitters. Most samples were taken from chicks just fed $(n=18)$, but 3 were collected from adults directly.

Commercial fisheries. On the Kerguelen shelf, 3 types of legal fishing vessels operated during February 1994 and 1995 (Fig. 1). These were: (1) 3 Ukrainian longliners ('Nikolay Reshetnyak', 'Pantikopey' and 'Primoretz') targetting Patagonian toothfish Dissostichus eleginoides mainly in the western part of the

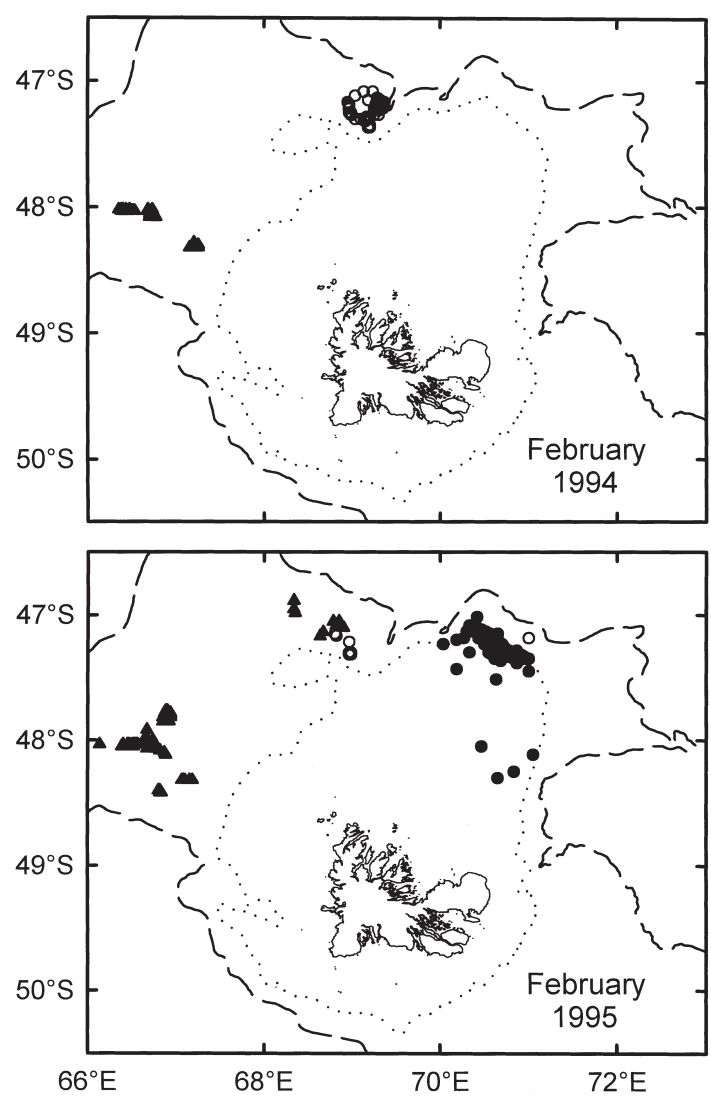

Fig. 1. Diomedea melanophrys. Location of longline $(\boldsymbol{\Lambda})$ and trawl (O) fisheries for Patagonian toothfish Dissostichus eleginoides, and of the trawl fishery targetting icefish Champsocephalus gunnari (•) in February 1994 and 1995 in Kerguelen waters. Isobaths 200 and $1000 \mathrm{~m}$ are indicated 
shelf. Longlines were set along the shelf-break at depths of 400 to $650 \mathrm{~m}$ and the hooks were baited with horse mackerel Trachurus trachurus capensis. Homogenised offal from processed $D$. eleginoides was discharged from the opposite side of the vessel from the line setting, providing potential food for seabirds. Fish bycatch comprised mainly large skates that birds could not handle before they sank (Cherel et al. 1996). (2) One French trawler ('Kerguelen de Tremarec') targetting $D$. eleginoides. Bottom trawling was concentrated in the northern part of the shelf at depths of 350 to $750 \mathrm{~m}$. Large-sized offal (head, gut, bones, tail) was released, but were difficult to swallow because they were not homogenized. Few fish and squid occurred as bycatch (Weimerskirch et al. 2000). (3) Three Ukrainian trawlers ('Mys Ostrovskogo', 'Tchatyr Dag' and 'Vorodoznoye') targetting mackerel icefish Champsocephalus gunnari in water 170 to $240 \mathrm{~m}$ deep in the northeastern shelf during February 1995. No offal was available to seabirds because icefish were frozen whole. Very few fish and squid occurred as bycatch (Weimerskirch et al. 2000).

To our knowledge, there were no illegal fishing vessels in 1994 and 1995, the first ones being observed in Kerguelen waters in 1997. Since that year, all the identified pirate vessels were longliners, not trawlers, targetting Dissostichus eleginoides. Fish bycatch by legal longliners includes large skates (see above) and other species rarely caught by albatrosses, such as Macrourus carinatus, M. holotrachys and Antimora rostrata (Duhamel et al. 1997). Moreover, the lack of hooks and bait remains in the birds' stomachs indicated little interaction between black-browed albatrosses and longliners (pirate or not) in 1994 and 1995 (see 'Discussion'). Consequently, it is unlikely that a significant part of the albatross diet was discards and offal thrown overboard from unknown poaching vessels during the study period.

Statistics. Data were statistically analysed using SYSTAT 7.0 for Windows (Wilkinson 1997). Values are means $\pm \mathrm{SD}$.

\section{RESULTS}

\section{Duration of foraging trips and meal size}

Trip duration of black-browed albatrosses, measured by visual observation of adults visiting nests, ranged from 0.2 to $11.4 \mathrm{~d}(\mathrm{n}=535)$ in February, with no significant differences between years $(2.0 \pm 1.5 \mathrm{~d}, \mathrm{n}=290$, vs $2.1 \pm 1.3 \mathrm{~d}, \mathrm{n}=245$, in 1994 and 1995, respectively, Student's $t$-test, $t=0.16, \mathrm{p}=0.871$ ).

The mean mass of 114 stomach samples obtained from chicks was $697 \mathrm{~g}$, a value heavier than meal size $(543 \pm 265 \mathrm{~g}, \mathrm{n}=350$, and $449 \pm 216 \mathrm{~g}, \mathrm{n}=284$, in 1994 and 1995, respectively), because chick samples included not only the last meal, but also items from previous feeds. Stomach contents of chicks comprised on

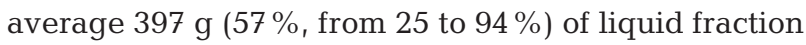
and $300 \mathrm{~g}(43 \%$, from 6 to $75 \%)$ of solid. Accumulated items, mainly cephalopod beaks and stones, formed $2.1 \mathrm{~g}(0.7 \%)$ of the solid fraction; the remaining $297.5 \mathrm{~g}$ $(99.3 \%)$ were fresh items (Table 1$)$.

Table 1. Diomedea melanophrys. Mass and composition of the food in stomach contents of chicks in 1994 and 1995 at Canyon des Sourcils Noirs, Kerguelen Islands

\begin{tabular}{|c|c|c|c|c|c|}
\hline & $\begin{array}{c}\text { Total } \\
(\mathrm{n}=114)\end{array}$ & $\begin{array}{c}1994 \\
(\mathrm{n}=45)\end{array}$ & $\begin{array}{c}1995 \\
(\mathrm{n}=69)\end{array}$ & \multicolumn{2}{|c|}{$\begin{array}{c}\text { Statistics } \\
\text { (1994 vs 1995) }\end{array}$} \\
\hline Mass (g) & $697 \pm 263$ & $733 \pm 288$ & $672 \pm 245$ & $t=1.21$ & $\mathrm{p}=0.229$ \\
\hline Liquid fraction (g) & $397 \pm 199$ & $462 \pm 233$ & $354 \pm 162$ & $t=2.91$ & $\mathrm{p}=0.004$ \\
\hline Solid fraction $(\mathrm{g})$ & $300 \pm 138$ & $271 \pm 125$ & $318 \pm 144$ & $t=1.78$ & $\mathrm{p}=0.078$ \\
\hline Fresh items $(\mathrm{g})$ & $297.5 \pm 137.2$ & $269.3 \pm 123.9$ & $315.9 \pm 143.2$ & $t=1.79$ & $\mathrm{p}=0.076$ \\
\hline Fish $(g)$ & $217.5 \pm 161.5$ & $186.9 \pm 128.2$ & $237.5 \pm 178.0$ & $U=1313$ & $\mathrm{p}=0.165$ \\
\hline Cephalopods (g) & $28.8 \pm 66.7$ & $30.0 \pm 69.2$ & $28.0 \pm 65.5$ & $U=1511$ & $\mathrm{p}=0.795$ \\
\hline Crustaceans (g) & $0.25 \pm 0.72$ & $0.07 \pm 0.30$ & $0.36 \pm 0.88$ & $U=1206$ & $\mathrm{p}=0.018$ \\
\hline Penguins (g) & $42.9 \pm 113.3$ & $45.2 \pm 112.9$ & $41.3 \pm 114.4$ & $U=1667$ & $\mathrm{p}=0.335$ \\
\hline Others $(\mathrm{g})$ & $8.0 \pm 42.8$ & $7.1 \pm 46.3$ & $8.6 \pm 40.7$ & $U=1383$ & $\mathrm{p}=0.120$ \\
\hline Accumulated beaks (n) & $10.9 \pm 15.8$ & $16.9 \pm 21.9$ & $7.1 \pm 8.1$ & $U=1863$ & $\mathrm{p}=0.071$ \\
\hline Parasitic nematods (n) & $10.7 \pm 13.9$ & $8.8 \pm 12.4$ & $12.0 \pm 14.8$ & $U=1266$ & $\mathrm{p}=0.096$ \\
\hline Stones (n) & $6.7 \pm 9.0$ & $4.8 \pm 7.4$ & $7.9 \pm 9.7$ & $U=1272$ & $\mathrm{p}=0.094$ \\
\hline \multicolumn{6}{|l|}{ Overall composition: } \\
\hline Fish (\%) & 73.1 & 69.4 & 75.2 & - & \\
\hline Cephalopods (\%) & 9.7 & 11.1 & 8.9 & - & \\
\hline Crustaceans (\%) & $<0.1$ & $<0.1$ & 0.1 & - & \\
\hline Penguins (\%) & 14.4 & 16.8 & 13.1 & - & \\
\hline Others (\%) & 2.7 & 2.7 & 2.7 & - & \\
\hline
\end{tabular}




\section{Diet}

In all the 114 samples pooled, chick food of blackbrowed albatrosses at Kerguelen during summer was dominated by fish, which accounted for $73.1 \%$ by fresh mass of the diet. Other significant food sources were penguin carrion (14.4\% by mass) and cephalopods $(9.7 \%)$, while crustaceans and other various organisms were only minor items ( $<0.1$ and $2.7 \%$, respectively) (Table 1). In terms of individual food samples, fish prevailed by fresh mass in $73.7 \%(\mathrm{n}=84)$ of the stomachs, penguin remains in $14.0 \%(\mathrm{n}=16)$, cephalopods in $8.8 \%(\mathrm{n}=10)$, and carrion of undetermined origin in $3.5 \%(\mathrm{n}=4)$ of the remaining samples.

A total of 817 fresh prey items from 58 species or prey categories were recovered from the 114 dietary samples (Table 2). Items included 433 (53.0\%) crustaceans, $232(28.7 \%)$ fishes and $82(10.0 \%)$ cephalopods. Crustaceans occurred in 40 samples (35.1\%) and dominated the diet by number but, owing to their small size, were of negligible mass. The 2 main crustacean species were the hyperiid amphipod Themisto gaudichaudii and the euphausiid Euphausia vallentini. Two other species also found in significant numbers were the isopod Serolis cornuta and the crab Halicarcinus planatus.

Fish, cephalopods and carrion occurred in 102 $(89.5 \%), 54(47.4 \%)$ and $29(25.4 \%)$ dietary samples. Excluding crustaceans, gastropods and polychaetes, which were predominantly secondary prey (see 'Discussion'), fish ranked first by number (67.4\%) and reconstituted mass $(75.3 \%)$, cephalopods ranked second (23.8 and $15.5 \%$, respectively), and carrion third (8.7 and $9.2 \%$ ). At the family level, albatross diet was dominated by nototheniid $(28.8 \%$ by number and $41.8 \%$ by mass) and channichthyid (19.8 and 19.6\%) fish, ommastrephid squid (16.9 and $12.2 \%$ ) and penguins (Spheniscidae, 6.4 and 8.0\%). Altogether, these

Table 2. Diomedea melanophrys. Frequency of occurrence, numbers and reconstituted mass of prey items identified from stomach contents during chick rearing (total for all 114 samples pooled)

\begin{tabular}{|c|c|c|c|c|c|c|}
\hline \multirow[t]{2}{*}{ Species } & \multicolumn{2}{|c|}{ Occurrence } & \multicolumn{2}{|c|}{ Number } & \multicolumn{2}{|c|}{ Reconstituted mass } \\
\hline & (n) & $(\%)$ & (n) & $(\%)$ & (g) & $(\%)$ \\
\hline \multicolumn{7}{|l|}{ Carrion } \\
\hline \multicolumn{7}{|l|}{ Aves } \\
\hline Unidentified Spheniscidae & 22 & 19.3 & 22 & 2.7 & 7257.2 & 8.0 \\
\hline Pachyptila desolata & 1 & 0.9 & 1 & 0.1 & 145.0 & 0.2 \\
\hline Unidentified carrion & 7 & 6.1 & 7 & 0.9 & 904.5 & 1.0 \\
\hline \multicolumn{7}{|l|}{ Fish } \\
\hline \multicolumn{7}{|l|}{ Rajidae } \\
\hline Bathyraja sp. & 16 & 14.0 & 16 & 2.0 & 4126.9 & 4.5 \\
\hline \multicolumn{7}{|l|}{ Paralepididae } \\
\hline Magnisudis prionosa & 1 & 0.9 & 1 & 0.1 & 112.6 & 0.1 \\
\hline \multicolumn{7}{|l|}{ Myctophidae } \\
\hline Krefftichthys anderssoni & 1 & 0.9 & 1 & 0.1 & 0.9 & $<0.1$ \\
\hline Protomyctophum bolini & 1 & 0.9 & 1 & 0.1 & 1.3 & $<0.1$ \\
\hline \multicolumn{7}{|l|}{ Muraenolepididae } \\
\hline Muraenolepis marmoratus/orangiensis & 14 & 12.3 & 15 & 1.8 & 2730.8 & 3.0 \\
\hline \multicolumn{7}{|l|}{ Moridae } \\
\hline Laemonema kongi & 1 & 0.9 & 1 & 0.1 & 21.0 & $<0.1$ \\
\hline \multicolumn{7}{|l|}{ Macrouridae } \\
\hline Macrourus carinatus/holotrachys & 3 & 2.6 & 3 & 0.4 & 2722.1 & 3.0 \\
\hline \multicolumn{7}{|l|}{ Melamphaidae } \\
\hline Melamphaidae sp. A & 1 & 0.9 & 1 & 0.1 & 31.3 & $<0.1$ \\
\hline \multicolumn{7}{|l|}{ Congiopodidae } \\
\hline Zanclorhynchus spinifer & 8 & 7.0 & 8 & 1.0 & 1025.8 & 1.1 \\
\hline \multicolumn{7}{|l|}{ Nototheniidae } \\
\hline Dissostichus eleginoides & 25 & 21.9 & 32 & 3.9 & 16658.2 & 18.3 \\
\hline Gobionotothen acuta & 2 & 1.8 & 4 & 0.5 & 81.6 & $<0.1$ \\
\hline Lepidonotothen squamifrons & 25 & 21.9 & 26 & 3.2 & 10519.3 & 11.6 \\
\hline Lepidonotothen mizops & 4 & 3.5 & 6 & 0.7 & 92.0 & 0.1 \\
\hline Notothenia cyanobrancha & 17 & 14.9 & 19 & 2.3 & 4066.7 & 4.5 \\
\hline Notothenia rossii & 4 & 3.5 & 4 & 0.5 & 4166.6 & 4.6 \\
\hline Paranotothenia magellanica & 3 & 2.6 & 3 & 0.4 & 304.2 & 0.3 \\
\hline Unidentified Nototheniidae & 5 & 4.4 & 5 & 0.6 & 1997.2 & 2.2 \\
\hline
\end{tabular}


Table 2 (continued)

\begin{tabular}{|c|c|c|c|c|c|c|}
\hline \multirow[t]{2}{*}{ Species } & \multicolumn{2}{|c|}{ Occurrence } & \multicolumn{2}{|c|}{ Number } & \multicolumn{2}{|c|}{ Reconstituted mas: } \\
\hline & (n) & $(\%)$ & (n) & $(\%)$ & $(g)$ & $(\%)$ \\
\hline \multicolumn{7}{|l|}{ Harpagiferidae } \\
\hline Harpagifer spinosus & 4 & 3.5 & 4 & 0.5 & 14.3 & $<0.1$ \\
\hline \multicolumn{7}{|l|}{ Channichthyidae } \\
\hline Champsocephalus gunnari & 12 & 10.5 & 19 & 2.3 & 1899.9 & 2.1 \\
\hline Channichthys rhinoceratus & 40 & 35.1 & 46 & 5.6 & 15314.2 & 16.9 \\
\hline Unidentified Channichthyidae & 3 & 2.6 & 3 & 0.4 & 549.3 & 0.6 \\
\hline \multicolumn{7}{|l|}{ Gempylidae } \\
\hline Paradiplospinus gracilis & 1 & 0.9 & 1 & 0.1 & 40.5 & $<0.1$ \\
\hline \multicolumn{7}{|l|}{ Achiropsettidae } \\
\hline Mancopsetta maculata & 1 & 0.9 & 1 & 0.1 & 231.2 & 0.3 \\
\hline Unidentified fish & 11 & 9.6 & 12 & 1.5 & 1610.3 & 1.8 \\
\hline \multicolumn{7}{|l|}{ Cephalopoda } \\
\hline \multicolumn{7}{|l|}{ Ommastrephidae } \\
\hline Todarodes sp. & 21 & 18.4 & 39 & 4.8 & 6892.1 & 7.6 \\
\hline Martialia hyadesi & 9 & 7.9 & 14 & 1.7 & 3275.5 & 3.6 \\
\hline Unidentified Ommastrephidae & 5 & 4.4 & 5 & 0.6 & 914.2 & 1.0 \\
\hline \multicolumn{7}{|l|}{ Onychoteuthidae } \\
\hline Moroteuthis ingens & 1 & 0.9 & 1 & 0.1 & 415.7 & 0.5 \\
\hline Kondakovia longimana & 1 & 0.9 & 1 & 0.1 & 160.9 & 0.2 \\
\hline \multicolumn{7}{|l|}{ Mastigoteuthidae } \\
\hline Mastigoteuthis psychrophila & 1 & 0.9 & 1 & 0.1 & 31.0 & $<0.1$ \\
\hline \multicolumn{7}{|l|}{ Octopodidae } \\
\hline Benthoctopus thielei & 17 & 14.9 & 20 & 2.5 & 2181.6 & 2.4 \\
\hline Unidentified Cephalopoda & 1 & 0.9 & 1 & 0.1 & 197.0 & 0.2 \\
\hline \multicolumn{7}{|l|}{ Gastropoda } \\
\hline Margarella expansa & 1 & 0.9 & 23 & 2.8 & 3.7 & $<0.1$ \\
\hline \multicolumn{7}{|l|}{ Polychaeta } \\
\hline Polynoidae sp. & 11 & 9.6 & 17 & 2.1 & 17.0 & $<0.1$ \\
\hline \multicolumn{7}{|l|}{ Crustacea } \\
\hline Euphausiacea & & & & & & \\
\hline Euphausia vallentini & 2 & 1.8 & 69 & 8.5 & 5.0 & $<0.1$ \\
\hline Euphausia sp. & 3 & 2.6 & 5 & 0.6 & 0.4 & $<0.1$ \\
\hline Caridea & & & & & & \\
\hline Pasiphaea scotiae & 1 & 0.9 & 2 & 0.2 & 4.5 & $<0.1$ \\
\hline Reptantia & & & & & & \\
\hline Halicarcinus planatus & 7 & 6.1 & 19 & 2.3 & 39.1 & $<0.1$ \\
\hline Mysida & & & & & & \\
\hline Neognathophausia ingens & 1 & 0.9 & 1 & 0.1 & 7.5 & $<0.1$ \\
\hline Neognathophausia gigas & 1 & 0.9 & 1 & 0.1 & 7.5 & $<0.1$ \\
\hline Isopoda & & & & & & \\
\hline Arcturides cornutus & 1 & 0.9 & 1 & 0.1 & $<0.1$ & $<0.1$ \\
\hline Antarcturus sp. & 3 & 2.6 & 5 & 0.6 & 1.1 & $<0.1$ \\
\hline Serolis septemcarinata & 1 & 0.9 & 1 & 0.1 & $<0.1$ & $<0.1$ \\
\hline Serolis cornuta & 6 & 5.3 & 29 & 3.6 & 11.6 & $<0.1$ \\
\hline Serolis latifrons & 1 & 0.9 & 1 & 0.1 & 1.6 & $<0.1$ \\
\hline Aega semicarinata & 2 & 1.8 & 2 & 0.2 & 3.5 & $<0.1$ \\
\hline Cassidinopsis emarginata & 3 & 2.6 & 4 & 0.5 & 1.0 & $<0.1$ \\
\hline Amphipoda & & & & & & \\
\hline Themisto gaudichaudii & 22 & 19.3 & 281 & 34.4 & 25.6 & $<0.1$ \\
\hline Cyllopus magellanicus & 1 & 0.9 & 1 & 0.1 & 0.1 & $<0.1$ \\
\hline Vibilia antarctica & 1 & 0.9 & 1 & 0.1 & $<0.1$ & $<0.1$ \\
\hline Jassa cf. justi & 1 & 0.9 & 1 & 0.1 & $<0.1$ & $<0.1$ \\
\hline Uristes gigas & 2 & 1.8 & 2 & 0.2 & 0.3 & $<0.1$ \\
\hline Unidentified Gammaridea & 3 & 2.6 & 5 & 0.6 & 0.7 & $<0.1$ \\
\hline Copepoda & & & & & & \\
\hline Lophoura sp. & 1 & 0.9 & 1 & 0.1 & 0.7 & $<0.1$ \\
\hline Unidentified Pennellidae/Sphyriidae & 1 & 0.9 & 1 & 0.1 & 0.7 & $<0.1$ \\
\hline Total & - & - & 817 & 100.0 & 90824.2 & 100.0 \\
\hline
\end{tabular}


4 families accounted for $71.8 \%$ of the number of prey and $81.6 \%$ of the reconstituted mass of the diet.

Black-browed albatrosses fed upon a large diversity of fish ( 21 species). Only 1 species constituted $>10 \%$ of the number of prey (excluding secondary items), the channichthyid Channichthys rhinoceratus (13.4\%). Three species constituted $>10 \%$ of the reconstituted mass: Dissostichus eleginoides (18.3\%), C. rhinoceratus $(16.9 \%)$ and Lepidonotothen squamifrons (11.6\%). Noticeable was also the occurrence of skates Bathyraja sp. ( $4.7 \%$ by number and $4.5 \%$ by mass), and the bony fishes Muraenolepis marmoratus and Muraenolepis marmoratus/orangiensis (4.4 and 3.0\%), Notothenia cyanobrancha (5.5 and $4.5 \%$ ), and Champsocephalus gunnari (5.5 and $2.1 \%$ ). Seven fish species were occasionally eaten (each with 3 to 8 individuals), and 7 species were rare prey items, being found only once in the samples (Table 2).

Three cephalopods were important prey of albatrosses in summer, the ommastrephid squids Todarodes sp. ( $11.3 \%$ by number and $7.6 \%$ by mass), probably Todarodes angolensis (Cherel \& Weimerskirch 1995), and Martialia hyadesi (4.1 and 3.6\%), and the octopus Benthoctopus thielei (5.8 and 2.4\%). Three other species were rare prey items (Table 2). Analysis of a much larger number of beaks (573 lower and 652

Table 3. Diomedea melanophrys. Cephalopod diet of black-browed albatrosses in 1994 (n = 45) and 1995 (n = 69) at Canyon des Sourcils Noirs, Kerguelen Islands. Values are number, and the percentage contribution by number, of accumulated lower and upper beaks

\begin{tabular}{|c|c|c|c|c|c|c|}
\hline \multirow[t]{2}{*}{ Species } & \multicolumn{3}{|c|}{ - Lower beaks - } & \multicolumn{3}{|c|}{ - Upper beaks } \\
\hline & $\begin{array}{l}\text { Total } \\
\mathrm{n}(\%)\end{array}$ & $\begin{array}{c}1994 \\
\mathrm{n}(\%)\end{array}$ & $\begin{array}{l}1995 \\
\text { n (\%) }\end{array}$ & $\begin{array}{l}\text { Total } \\
\mathrm{n}(\%)\end{array}$ & $\begin{array}{c}1994 \\
\text { n }(\%)\end{array}$ & $\begin{array}{c}1995 \\
\text { n (\%) }\end{array}$ \\
\hline \multicolumn{7}{|l|}{ Teuthoidea } \\
\hline \multicolumn{7}{|l|}{ Ommastrephidae } \\
\hline Todarodes sp. & $52(9.1)$ & $27(8.1)$ & $25(10.5)$ & $4(0.6)^{\mathrm{a}}$ & $2(0.5)^{\mathrm{a}}$ & $2(0.8)^{\mathrm{a}}$ \\
\hline Martialia hyadesi & $32(5.6)$ & $31(9.3)$ & $1(0.4)$ & $0^{a}$ & $0^{\mathrm{a}}$ & $0^{\mathrm{a}}$ \\
\hline Ommastrephidae sp. & $183(31.9)^{b}$ & $98(29.3)^{b}$ & $85(35.7)^{b}$ & $326(50.0)^{\mathrm{c}}$ & $204(49.4)^{\mathrm{c}}$ & $122(51.0)^{\mathrm{c}}$ \\
\hline \multicolumn{7}{|l|}{ Onychoteuthidae } \\
\hline Moroteuthis ingens & $4(0.7)$ & $2(0.6)$ & $2(0.8)$ & $4(0.6)$ & $1(0.2)$ & $3(1.3)$ \\
\hline Moroteuthis knipovitchi & $3(0.5)$ & $2(0.6)$ & $1(0.4)$ & $1(0.2)$ & $1(0.2)$ & 0 \\
\hline Moroteuthis sp. B (Imber) & $4(0.7)$ & $1(0.3)$ & $3(1.3)$ & $4(0.6)$ & $2(0.5)$ & $2(0.8)$ \\
\hline Kondakovia longimana & $19(3.3)$ & $5(1.5)$ & $14(5.9)$ & $16(2.5)$ & $4(1.0)$ & $12(5.0)$ \\
\hline \multicolumn{7}{|l|}{ Gonatidae } \\
\hline Gonatus antarcticus & $4(0.7)$ & $3(0.9)$ & $1(0.4)$ & $7(1.1)$ & $6(1.5)$ & $1(0.4)$ \\
\hline \multicolumn{7}{|l|}{ Octopoteuthidae } \\
\hline Taningia danae & $3(0.5)$ & $2(0.6)$ & $1(0.4)$ & $2(0.3)$ & $2(0.5)$ & 0 \\
\hline \multicolumn{7}{|l|}{ Histioteuthidae } \\
\hline Histioteuthis atlantica & $2(0.3)$ & 0 & $2(0.8)$ & $2(0.3)$ & $1(0.2)$ & $1(0.4)$ \\
\hline \multicolumn{7}{|l|}{ Neoteuthidae } \\
\hline Alluroteuthis antarcticus & $1(0.2)$ & 0 & $1(0.4)$ & $2(0.3)$ & 0 & $2(0.8)$ \\
\hline Nototeuthis dimegacotyle & $3(0.5)$ & $1(0.3)$ & $2(0.8)$ & $2(0.3)$ & $2(0.5)$ & 0 \\
\hline \multicolumn{7}{|l|}{ Mastigoteuthidae } \\
\hline Mastigoteuthis psychrophila & $3(0.5)$ & $1(0.3)$ & $2(0.8)$ & $1(0.2)$ & $1(0.2)$ & 0 \\
\hline Mastigoteuthis sp. & $1(0.2)$ & $1(0.3)$ & 0 & 0 & 0 & 0 \\
\hline \multicolumn{7}{|l|}{ Chiroteuthidae } \\
\hline Chiroteuthis veranyi & $3(0.5)$ & $2(0.6)$ & $1(0.4)$ & $9(1.4)$ & $2(0.5)$ & $7(2.9)$ \\
\hline \multicolumn{7}{|l|}{ Batoteuthidae } \\
\hline Batoteuthis skolops & $6(1.0)$ & $5(1.5)$ & $1(0.4)$ & $18(2.8)$ & $18(4.4)$ & 0 \\
\hline \multicolumn{7}{|l|}{ Cranchiidae } \\
\hline Galiteuthis glacialis & $116(20.2)$ & $88(26.3)$ & $28(11.8)$ & $131(20.1)$ & $104(25.2)$ & $27(11.3)$ \\
\hline \multicolumn{7}{|l|}{ Octopoda } \\
\hline \multicolumn{7}{|l|}{ Octopodidae } \\
\hline Benthoctopus thielei ${ }^{\mathrm{d}}$ & $134(23.4)$ & $66(19.7)$ & $68(28.6)$ & $123(18.9)$ & $63(15.3)$ & $60(25.1)$ \\
\hline Total & 573 & 335 & 238 & 652 & 413 & 239 \\
\hline Unidentifiable beaks (eroded) & 7 & 4 & 3 & 14 & 7 & 7 \\
\hline \multicolumn{7}{|c|}{$\begin{array}{l}\text { a Upper beaks of adults from Todarodes sp. and Martialia hyadesi } \\
\text { b'Eroded lower beaks of juveniles from Todarodes sp. and Martialia hyadesi } \\
{ }^{c} \text { Eroded and intact upper beaks of juveniles from Todarodes sp. and Martialia hyadesi } \\
\text { de Possibly including some Graneledone gonzalezi }\end{array}$} \\
\hline
\end{tabular}


upper beaks) that were accumulated in the chicks' stomach showed a more diverse cephalopod diet, including 16 different species of squids and 1 species of octopus. Accumulated beaks nevertheless confirmed the predominance of ommastrephids $(48.7 \%$ of the total number of beaks), and of B. thielei $(21.0 \%)$ in the albatross diet (Table 3). They added a new important prey species to the albatross diet, the cranchiid Galiteuthis glacialis $(20.2 \%)$, that was found once (2 specimens) as fresh items in an incomplete stomach content. Altogether, the 4 dominant cephalopod species accounted for $89.9 \%$ of the number of accumulated beaks. Other significant squid prey were Kondakovia longimana (2.9\%) and Batoteuthis skolops (2.0\%).

It was not possible to identify penguins at the species level, but one albatross chick was unable to swallow a leg of macaroni penguin Eudyptes chrysolophus during the study period. In 7 cases, a large piece of skin with blubber still attached was found. In 6 of them, the plumage showed new feathers growing under the skin, clearly indicating that penguins were in active moult, in the phase of feather synthesis which begins at sea (Cherel et al. 1994).

\section{Prey size}

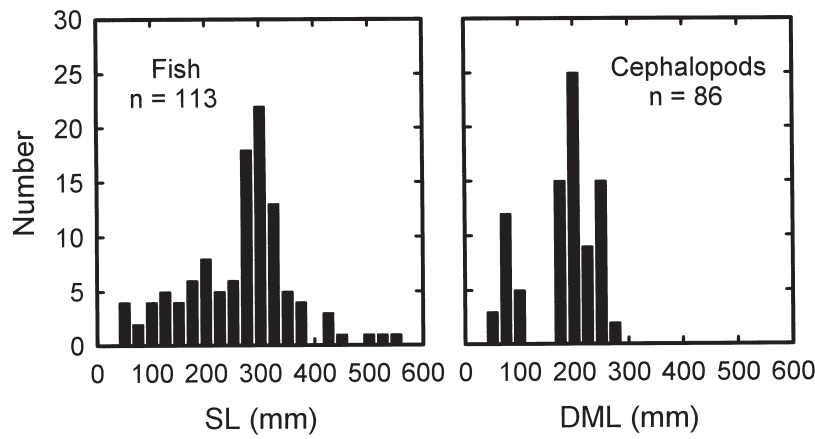

Fig. 2. Diomedea melanophrys. Numerical distribution of fish standard length (SL) and cephalopod dorsal mantle length (DML) of fresh prey found in chick stomach contents at Kerguelen Islands in summer
Estimated sizes of fish averaged $250 \pm 98 \mathrm{~mm}(\mathrm{n}=$ 113) with a main mode at 275 to $300 \mathrm{~mm}$ (Fig. 2). Fish ranged in size from $43 \mathrm{~mm}$ SL (Harpagifer spinosus) to $529 \mathrm{~mm}$ SL (Macrourus carinatus/holotrachys). Estimated fish masses averaged $292 \pm 330 \mathrm{~g}$ and ranged from $0.9 \mathrm{~g}$ (Krefftichthys anderssoni) to $2012 \mathrm{~g}$ (Dissostichus eleginoides) (Table 4). Remains of larger $D$. eleginoides were found, but the lack of otoliths and dentary bones precluded an estimation of size. Only 1 size-class of Lepidonothen squamifrons was observed with a mode at $26 \mathrm{~mm}$ mandible length (ML) (SL = $294 \mathrm{~mm}, \mathrm{M}=480 \mathrm{~g}$ ), while Channichthys rhinoceratus of different sizes were eaten (main mode at $48 \mathrm{~mm} \mathrm{ML}$, $\mathrm{SL}=295$ mm, M = 259 g) (Fig. 3).

Table 4. Diomedea melanophrys. Measured fish mandible length (ML), otolith length (OL), squid lower rostral length (LRL) and octopus lower hood length (LHL), and estimated fish standard length (SL), squid dorsal mantle length (DML) and body mass (M) of the main prey identified from stomach contents during chick rearing. Values are means \pm SD with ranges in parentheses

\begin{tabular}{|c|c|c|c|c|c|}
\hline \multirow[t]{2}{*}{ Species } & \multicolumn{3}{|c|}{ Measured parameters (mm) } & \multirow[t]{2}{*}{ SL/DML (mm) } & \multirow[t]{2}{*}{$M(g)$} \\
\hline & $\mathrm{n}$ & Parameters & S Values & & \\
\hline \multicolumn{6}{|l|}{ Fish } \\
\hline Bathyraja spp. & 1 & - & - & 370 & 274 \\
\hline Muraenolepis marmoratus/orangiensis & 8 & ML & $22 \pm 7(7-30)$ & $264 \pm 37(184-305)$ & $174 \pm 55(66-246)$ \\
\hline Zanclorhynchus spinifer & 5 & ML & $15 \pm 2(11-18)$ & $177 \pm 28(136-209)$ & $132 \pm 65(49-218)$ \\
\hline Dissostichus eleginoides & 4 & OL & $8.9 \pm 0.6(8.3-9.6)$ & $466 \pm 43(424-515)$ & $1499 \pm 436(1083-2012)$ \\
\hline Gobionotothen acuta & 4 & OL & $3.9 \pm 0.9(2.8-4.7)$ & $124 \pm 33(83-153)$ & $20 \pm 15(4-35)$ \\
\hline Lepidonotothen squamifrons & 16 & ML & $25 \pm 2(21-28)$ & $278 \pm 20(238-316)$ & $408 \pm 95(238-611)$ \\
\hline Notothenia cyanobrancha & 14 & OL & $4.2 \pm 0.7(2.7-4.9)$ & $224 \pm 51(114-280)$ & $214 \pm 112(23-373)$ \\
\hline Notothenia rossii & 3 & ML & $26 \pm 8(18-33)$ & $347 \pm 65(276-404)$ & $919 \pm 498(396-1388)$ \\
\hline Paranotothenia magellanica & 3 & ML & $16 \pm 2(14-18)$ & $188 \pm 24(168-215)$ & $101 \pm 39(71-145)$ \\
\hline Harpagifer spinosus & 4 & $\mathrm{OL}$ & $2.4 \pm 0.5(1.9-3.0)$ & $50 \pm 7(43-59)$ & $3.6 \pm 1.7(1.9-5.7)$ \\
\hline Champsocephalus gunnari & 7 & ML & $25 \pm 5(17-31)$ & $219 \pm 44(153-277)$ & $93 \pm 59(23-183)$ \\
\hline Channichthys rhinoceratus & 30 & ML & $50 \pm 8(30-67)$ & $305 \pm 42(195-403)$ & $331 \pm 173(45-933)$ \\
\hline \multicolumn{6}{|l|}{ Cephalopods $^{\text {a }}$} \\
\hline Todarodes angolensis & 109 & LRL & $5.0 \pm 1.1(3.8-10.6)$ & $196 \pm 44(144-428)$ & $239 \pm 253(93-1758)$ \\
\hline Martialia hyadesi & 42 & LRL & $4.6 \pm 0.6(3.9-7.9)$ & $239 \pm 18(218-336)$ & $248 \pm 81(175-716)$ \\
\hline Kondakovia longimana & 17 & LRL 11. & $1.9 \pm 3.0(4.6-16.3)$ & $423 \pm 111(149-586)$ & $2071 \pm 1135(87-4720)$ \\
\hline Galiteuthis glacialis & 132 & LRL & $5.2 \pm 0.3(4.3-5.9)$ & $225 \pm 12(188-251)$ & $99 \pm 13(64-130)$ \\
\hline Benthoctopus thielei & 117 & LHL & $4.9 \pm 1.2(1.7-8.4)$ & $69 \pm 15(29-113)$ & $130 \pm 71(7-504)$ \\
\hline
\end{tabular}



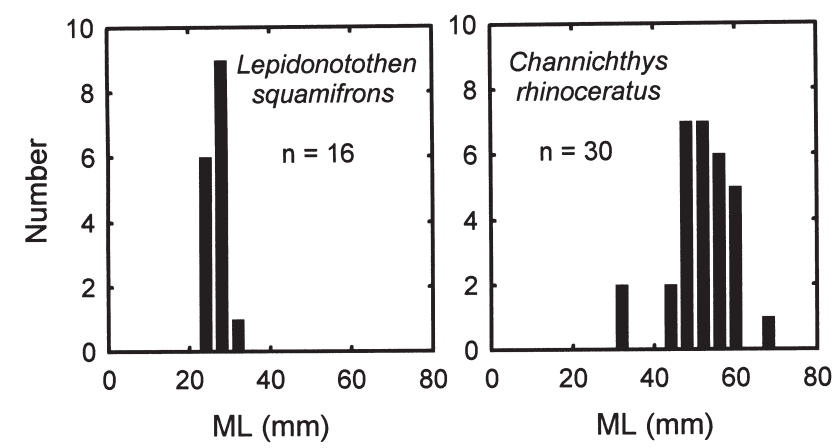

Fig. 3. Diomedea melanophrys. Number of lower mandibles (dentary bones) of the nototheniid fish Lepidonotothen squamifrons and the channichthyid Channichthys rhinoceratus in stomach contents at Kerguelen Islands during summer as a function of mandible length (ML)

Estimated sizes of fresh cephalopods averaged $168 \pm$ $62 \mathrm{~mm}(\mathrm{n}=86)$ with 3 modes at 50 to 75,175 to 200 and 225 to $250 \mathrm{~mm}$, respectively (Fig. 2). They ranged from $29 \mathrm{~mm}$ DML (Benthoctopus thielei) to $254 \mathrm{~mm}$ DML (Martialia hyadesi). Estimated cephalopod masses averaged $176 \pm 67 \mathrm{~g}$ and ranged from $7.2 \mathrm{~g}$ (B. thielei) to $416 \mathrm{~g}$ (Moroteuthis ingens). When taking into account the accumulated beaks, the largest prey eaten by black-browed albatrosses was a Taningia danae with an estimated DML and M of $745 \mathrm{~mm}$ and $7168 \mathrm{~g}$, respectively. Birds preyed mainly upon juvenile ommastrephids (beaks with undarkened wings) with a single mode at $4.8 \mathrm{~mm} \mathrm{LRL} \mathrm{(DML}=185 \mathrm{~mm}, \mathrm{M}=180 \mathrm{~g}$ ) and $4.3 \mathrm{~mm}$ LRL (DML = $227 \mathrm{~mm}, \mathrm{M}=204 \mathrm{~g}$ ) for Todarodes sp. and M. hyadesi, respectively (Fig. 4). They caught adults (wholly keratinized beaks) of Galiteuthis glacialis with a mode at $5.3 \mathrm{~mm}$ LRL (DML $=226 \mathrm{~mm}$, $\mathrm{M}=100 \mathrm{~g}$ ), and 2 size-classes of $B$. thielei with modes at $2.8 \mathrm{~mm}$ LHL (DML $=42 \mathrm{~mm}, \mathrm{M}=25 \mathrm{~g}$ ) and $5.3 \mathrm{~mm}$ LHL (DML = 73 mm, M = 142 g), respectively (Fig. 4).

\section{Temporal variations in diet}

No significant interannual differences were found in the mean mass of the main broad prey classes including fish, crustaceans and penguin carrion per dietary sample. Consequently, no changes occurred in the overall composition of the food between summer 1994 and summer 1995, with fish ranking first, penguins second and cephalopods third during the $2 \mathrm{yr}$ (Table 1).

The relative numbers of some of the main fresh prey (excluding secondary prey items) varied significantly between years. No fresh specimens of juvenile Martialia hyadesi occurred in summer 1995, and only 1 accumulated beak from an adult specimen was found at this time $\left(\chi^{2}{ }_{1}=20.59, \mathrm{p}<0.0001\right)$. The cephalopod diet in February 1995 was also marked by fewer Galiteuthis glacialis $\left(\chi^{2}{ }_{1}=18.13, \mathrm{p}<0.0001\right)$, more Benthoctopus thielei $\left(\chi^{2}{ }_{1}=6.11, \mathrm{p}=0.013\right)$ and Kondakovia longimana $\left(\chi^{2}{ }_{1}=8.36, \mathrm{p}=0.004\right)$, and no variation in the proportion of Todarodes $\mathrm{sp} .\left(\chi^{2}{ }_{1}=\right.$ 1.01, $\mathrm{p}=0.315)$. The size of juvenile Todarodes sp. was larger in 1994 than in 1995 (LRL: $5.2 \pm 1.0 \mathrm{~mm}$, $\mathrm{n}=37$, vs $4.7 \pm 0.4 \mathrm{~mm}, \mathrm{n}=68 ; t=3.94, \mathrm{p}=0.0001)$, but no interannual variations in size were observed for $G$. glacialis $(t=0.66, \mathrm{p}=0.514)$ and $B$. thielei $(t=1.22$, $\mathrm{p}=0.227$ ).

Summer 1994 showed a greater proportion of Dissostichus eleginoides (18.8 vs $4.4 \%$ by number in 1994 and 1995, respectively; Pearson Chi-square test, $\left.\chi_{1}^{2}=18.97, \mathrm{p}<0.0001\right)$, and summer 1995 showed more Notothenia cyanobrancha $\left(0.9\right.$ vs $7.9 \%$; $\chi_{1}^{2}=$ $7.41, \mathrm{p}=0.007$ ), Channichthys rhinoceratus (7.7 vs $16.3 \% ; \chi^{2}{ }_{1}=4.94, \mathrm{p}=0.026$ ) and Todarodes sp. (5.1 vs $\left.14.5 \% ; \chi^{2}{ }_{1}=6.80, \mathrm{p}=0.009\right)$. No interannual changes in length occurred for Muraenolepis marmoratus/ orangiensis (Mann-Whitney $U$-test, $U=9, \mathrm{p}=0.289$ ), Lepidonotothen squamifrons $(U=25, \mathrm{p}=0.777)$ and Champsocephalus gunnari $(U=4, \mathrm{p}=0.699)$, but $C$. rhinoceratus were larger in 1994 (ML: $53.3 \pm 4.0 \mathrm{~mm}$, $\mathrm{n}=8$, vs $47.6 \pm 10.0 \mathrm{~mm}, \mathrm{n}=22$ in $1995 ; U=130, \mathrm{p}=$ $0.049)$.
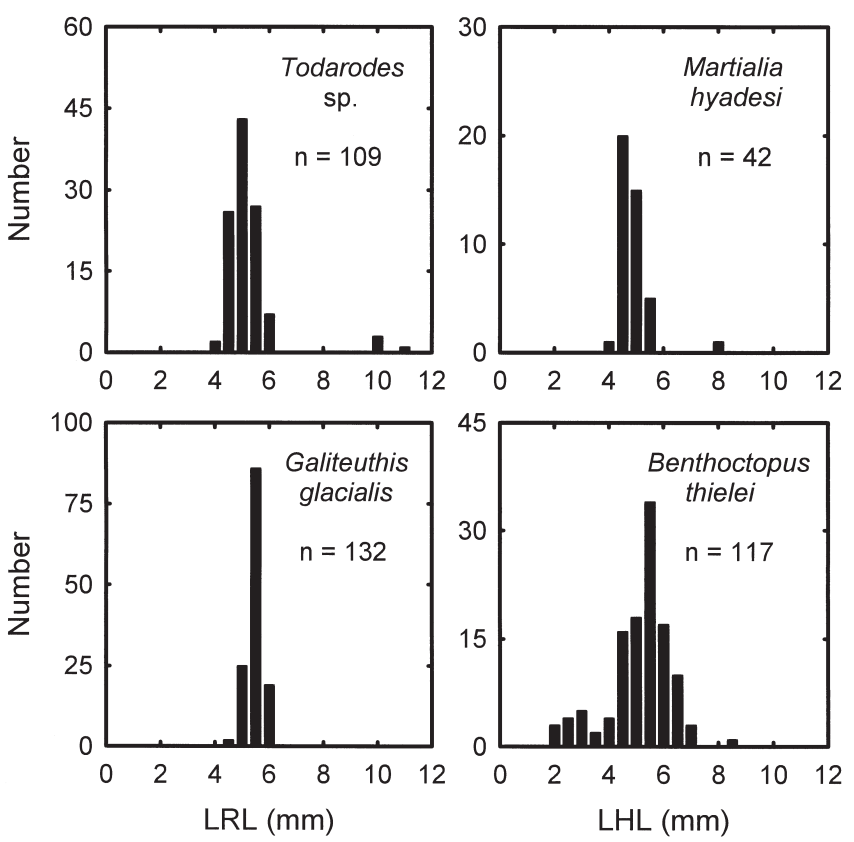

Fig. 4. Diomedea melanophrys. Number of fresh and accumulated lower beaks of the main cephalopod prey in stomach contents at Kerguelen Islands during summer as a function of lower rostral length (LRL) for squids and lower hood length (LHL) for the octopus Benthoctopus thielei. Note different scales of ordinates 

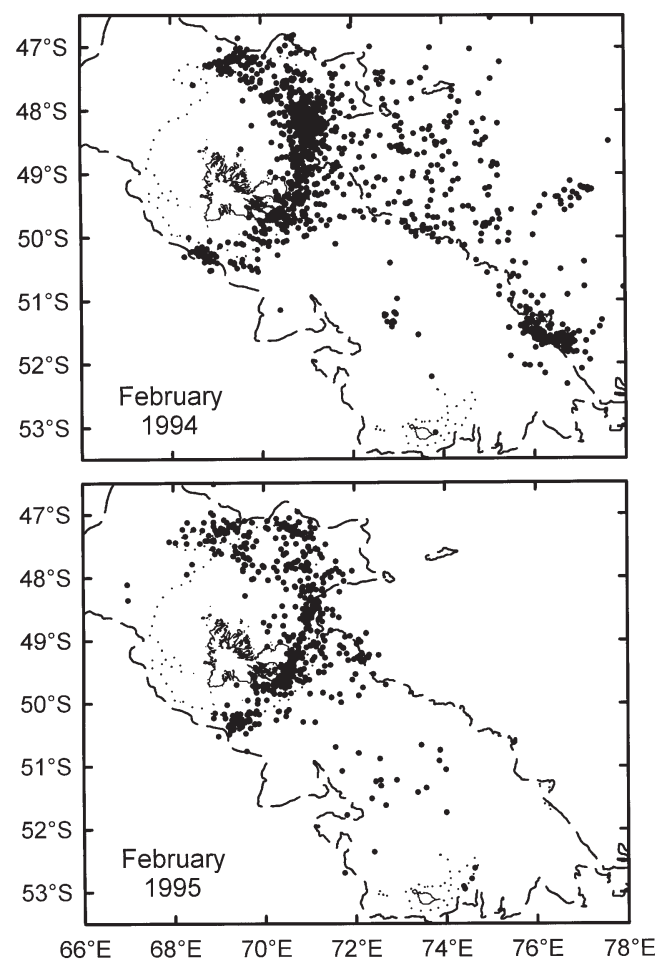

Fig. 5. Diomedea melanophrys. Distribution of all satellite locational fixes from Kerguelen Islands during the chick-rearing period in February 1994 (1290 fixes) and February 1995

\section{Satellite tracking}

In February 1994 and 1995, the duration of foraging trips of black-browed albatrosses fitted with transmitters ranged from 0.2 to $8.5 \mathrm{~d}(\mathrm{n}=53)$. Satellite locations indicated that, during these trips, the birds foraged mainly over the outer shelf and inner shelf-break of the Kerguelen Islands (Fig. 5). The maps clearly indicate that albatrosses foraged in northern, eastern and southern waters, but never on the western Kerguelen shelf. Four areas showed large numbers of fixes: first, along the coast near to the breeding colony; second, a large area on the inner shelf break in the north-northeast; third, a small area south of the archipelago; and fourth, the Shell Bank situated $480 \mathrm{~km}$ southeast from the colony. No major annual differences occurred, but albatrosses did move more to oceanic waters and to the Shell Bank in February 1994, and they foraged more on the northern edge of the shelf in February 1995. The maximum distance between the colony and the foraging zone was similar for both the $2 \mathrm{yr}(253 \pm 123$ vs $241 \pm 103 \mathrm{~km}$ in 1994 and 1995, respectively). Birds performed mostly commuting trips, which can be defined as follows. They first left the colony and moved in a more or less straight line to a specific area. They then remained in this area where they probably searched for food and fed. Finally, they returned
When comparing diet composition in February (both years pooled) with composition later in the season (early April 1994 and late March 1995), the only significant change was the larger amount of penguin carrion eaten by albatrosses in late chickrearing $(32.5 \pm 93.5 \mathrm{~g}, \mathrm{n}=84$, vs $72.0 \pm$ $154.3 \mathrm{~g}, \mathrm{n}=30 ; U=1026, \mathrm{p}=0.029)$. Consequently, penguins accounted for a higher proportion by mass at that time (11.4 vs $21.5 \%$ in February and March/April, respectively). Noticeable at the prey level was the lack of juvenile ommastrephids as fresh items in March/April, all Martialia hyadesi occurring in February 1994 only, and all Todarodes sp. in February (15.9 vs $0.0 \% ; \chi^{2}{ }_{1}=$ $17.52, \mathrm{p}<0.0001)$. Conversely, Benthoctopus thielei (2.8 vs $13.3 \% ; \chi_{1}^{2}=13.90$, $\mathrm{p}<0.0001$ ), and among fish, Dissostichus eleginoides ( 7.3 vs $14.3 \% ; \chi_{1}^{2}=4.03$, $\mathrm{p}=0.045)$ and Notothenia cyanobrancha ( 3.7 vs $\left.10.2 \% ; \chi^{2}{ }_{1}=5.75, p<0.016\right)$ were more abundant at this time, while a decrease in the importance of Lepidonotothen squamifrons $\left(10.2\right.$ vs $1.0 \% ; \chi^{2}{ }_{1}=8.38$, $\mathrm{p}=0.004$ ) was noted late in the season.

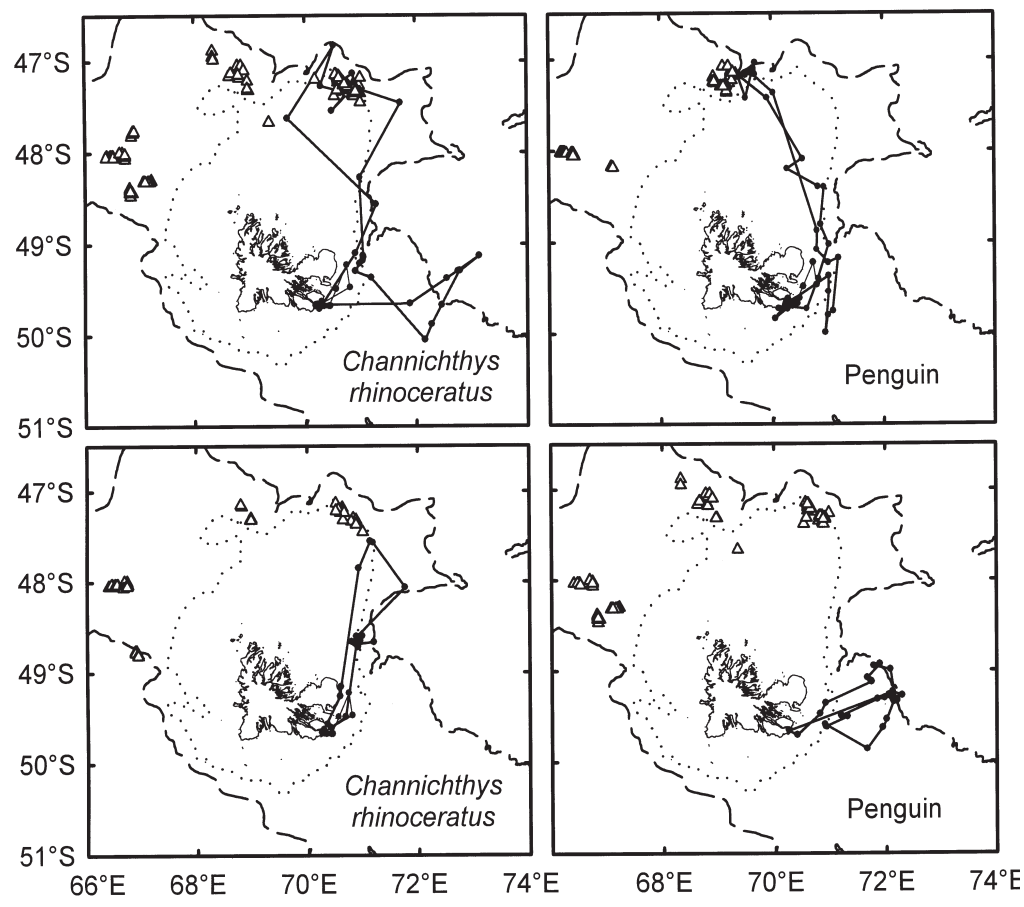

Fig. 6. Diomedea melanophrys. Detailed tracks of individual foraging trips during which adult birds fed on the fish Channichthys rhinoceratus and on penguin carrion. Location of the longlines and trawls performed during the tracks $(\Delta)$ and isobaths 200 and $1000 \mathrm{~m}$ are indicated 
rapidly to the colony to feed the single chick (Figs. 6 \& 7).

Excluding 6 samples, 15 stomach contents were associated with tracked foraging trips, and they contained various identified prey items. Of the 6 not furthermore included in the analysis, 1 contained accumulated items only, 2 were associated with an incomplete or no track of the foraging trip (battery failure), 2 contained unidentified ommastrephid squids, and 1 chick sample contained identified digested items not separable from the previous feed by the other parent.

Six samples contained Channichthys rhinoceratus, 5 samples Todarodes sp., 3 samples penguin remains and 2 samples contained skates (some samples contained more than 1 prey species). Other prey items were found only once in the stomach contents. Black-browed albatrosses fed on C. rhinoceratus and on penguins at several sites on the foraging grounds (Fig. 6). They caught the squid Todarodes sp. in the north-northeast area (Fig. 7). One bird preyed upon 3 Martialia hyadesi over the Shell Bank, and 2 other birds caught 1 Gonatus antarcticus and 1 Moroteuthis ingens in the northeast zone and while covering an extensive area in the south-southeast, respectively (Fig. 7). Among the 21 tracked foraging trips, 5 overlapped at least partially within the fishery zones. One albatross commuted to the area where a French trawler targetted Dissostichus eleginoides in February 1994. It subsequently fed its chick with penguin carrion (Fig. 6). Four birds foraged in the area where Ukrainian trawlers targetted Champsocephalus gunnari in February 1995. Two of these fed their chicks with $C$. rhinoceratus, and the 2 others fed theirs with Todarodes sp., and Todarodes sp. plus Benthoctopus thielei, respectively (Figs. 6 \& 7).

\section{DISCUSSION}

In summer 1994 and 1995, black-browed albatrosses rearing chicks at Kerguelen Islands foraged on the shelf and shelf-break where they fed mainly on fish. A large majority of specimens (85\%) were identified to species. This allowed the first precise qualitative (21 different species) and quantitative assessment of the fish diet for an albatross. The birds preyed mainly upon benthic and semipelagic nototheniids and channichthyids that were caught naturally. Fishery waste was only a minor component of the diet.

\section{Feeding on neritic prey}

The importance of fish ( $73 \%$ by fresh mass) in the diet agrees with an earlier study showing the predominance of fish in $87 \%$ of chick regurgitations collected in summer 1985 (Weimerskirch et al. 1988). These results are also in general agreement with diets determined at 2 other breeding localities, including South Georgia (27 to $73 \%$ by mass; Prince 1980, review in Cherel \& Klages 1998) and the Falkland Islands (26 to $82 \%$; Thompson 1992). It proves that the black-browed albatross is primarily a fish-eating species. A noticeable difference at Kerguelen was that penguins (mainly moulting birds) accounted for a significant part of albatross food $(14 \%)$, while penguin feathers were only noted as accumulated items elsewhere (Prince 1980, Ridoux 1994). At Crozet Islands, black-browed albatrosses have often been observed feeding on carcasses of penguins in inshore waters (Weimerskirch et al. 1986). It seems unlikely that albatrosses preyed upon healthy penguins, but they were able to scavenge on remains of penguins killed by other predators (Antarctic fur seals Arctocephalus gazella and killer whales Orcinus orca), or to kill penguins weakened by illness or, possibly, by the beginning of moult. It was not possible to identify penguins, but the most likely species to occur was the macaroni penguin Eudyptes chrysolophus because of its huge population at Ker- 
guelen (Weimerskirch et al. 1989). The fact that albatrosses fed more on penguins later in the season is also in agreement with the timing of moult in macaroni penguins. Moult of non-breeding birds begins in February and that of breeding adults in late March (Marchant \& Higgins 1990).

Black-browed albatrosses fed upon a large variety of fish, mostly nototheniids, channichthyids and muraenolepidids (Table 2). These 3 families comprise the few previously identified fish prey of albatrosses in the Southern Ocean (Cherel \& Klages 1998), including that of the black-browed albatross at South Georgia (Reid et al. 1996). The present work is the first to report skates and some endemic species of the Kerguelen Plateau (Gobionotothen acuta, Lepidonotothen mizops, Notothenia cyanobrancha, Channichthys rhinoceratus) in the diet of albatrosses (Cherel \& Klages 1998). Except for a few oceanic species (Magnisudis prionosa, myctophids, Paradiplospinus gracilis), which occurred in slope waters and further offshore (Duhamel 1998), all the fish species are characteristic of the shelf and upper shelf-break (Gon \& Heemstra 1990, Duhamel 1997). It is noteworthy that the common fishes in the albatross diet were species having the highest population biomasses there, i.e. Dissostichus eleginoides, Lepidonotothen squamifrons, Champsocephalus gunnari and C. rhinoceratus (Duhamel 1981)

Cephalopods, including the ommastrephids Todarodes sp. (probably $T$. angolensis, Cherel \& Weimerskirch 1995) and Martialia hyadesi, and Benthoctopus thielei accounted for $10 \%$ of the diet by fresh mass, a value within the range found previously for blackbrowed albatrosses at South Georgia and the Falklands. There they mainly feed on $M$. hyadesi and Loligo gahi, and Illex argentinus, respectively (Thompson 1992, Rodhouse \& Prince 1993). The present work therefore emphasizes the importance of juvenile ommastrephids in the diet of albatrosses (Cherel \& Klages 1998). It furthermore indicates, for the first time, the regular occurrence of an octopus species (the endemic $B$. thielei) in the diet of a small albatross, the only other known example being the larger royal albatross Diomedea epomophora feeding on Octopus cordiformis off New Zealand (Imber 1999). Accumulated items added another major squid prey to the black-browed albatross diet at Kerguelen, the cranchiid Galiteuthis glacialis (Table 3). It is striking to note that this species is common in the diet of many albatrosses from the Southern Ocean (Cherel \& Klages 1998), including the black-browed albatross at South Georgia (Rodhouse \& Prince 1993), but that soft parts were usually not found. The fairly large numbers of beaks of that species therefore suggests either that adult G. glacialis, an oceanic species (Nesis et al.
1998), was common in slope waters in spring and early summer at Kerguelen Islands, or alternatively, that birds foraged over more oceanic waters earlier in the breeding season.

Crustaceans were minor components of the food at Kerguelen Islands, but Antarctic krill Euphausia superba accounted for 5 to $42 \%$ of the diet by mass at South Georgia and lobster-krill Munida gregaria for $5 \%$ at the Falkland Islands (Prince 1980, Thompson 1992, review in Cherel \& Klages 1998). E. superba and $M$. gregaria do not occur in Kerguelen waters where the main crustacean items in albatross food were the subantarctic krill Euphausia vallentini and the pelagic amphipod Themisto gaudichaudii (Table 2). Most of them were probably secondarily ingested fish and penguin prey, for 2 reasons. First, they were found in association with channichthyids and nototheniids in dietary samples, and one penguin's stomach swallowed by an albatross was full of E. vallentini. Second, they are known to be major prey for neritic fish and penguins in Kerguelen/ Heard waters (Duhamel \& Hureau 1985, Green et al. 1998b, authors' unpubl. data). Other benthic crustaceans (isopods, gammariids, Halicarcinus planatus) were also probably secondary prey items, as were polychaetes, the gastropod Margarella expansa, and the small fish Harpagifer spinosus. All these benthic organisms occur commonly in the diet of Benthoctopus thielei, skates and bony fishes (Hureau 1970, Gon \& Heemstra 1990, authors' unpubl. data). The only crustaceans that were consequently primary prey of albatrosses were the oceanic Neognathophausia spp. and Pasiphaea scotiae, which have also been found in the diet of other procellariiform seabirds (Ridoux 1994, Cherel \& Klages 1998).

\section{Foraging over the shelf and slope waters}

The present study indicates that adult black-browed albatrosses rearing chicks from Canyon des Sourcils Noirs performed trips of short duration (about 2 d) during which they foraged almost exclusively over the outer shelf and inner slope waters of the northern, eastern and southern sectors of the Kerguelen Plateau. This is in agreement with previous observations at sea of dyed breeding individuals. In that case, birds from that colony fed essentially in the eastern and southern portions (Weimerskirch et al. 1988). Interestingly, albatrosses from another colony located in the north were observed not only in the northern but also in the western part of the shelf, where birds from Canyon des Sourcils Noirs never foraged. This suggests a spatial segregation at sea of individuals from different colonies (Weimerskirch et al. 1988), that can be linked to the 
broader context of intercolony competition for food in seabirds (Cairns 1992).

The habitat of the main prey (Table 5) is in agreement with blackbrowed albatrosses feeding in neriticslope waters, which is further emphasized by those food items associated with known foraging tracks (Figs. 6 $\& 7$ ). Some substantial changes in the prey composition of the food occurred between February 1994 and 1995, although the birds foraged essentially in the same areas. Albatrosses probably rely on resources that are predictable in location, but whose availability nevertheless varies from one year to the next. Site-fidelity in foraging seabirds has been demonstrated for several species from the northern hemisphere, and this behaviour is generally linked to physical processes that cause predictably located aggregations of prey in both time and space (Hunt et al. 1999).

Ship-based observations in the southern Indian Ocean confirm that the black-browed albatross is essentially a neritic-slope forager in summer, not only at Kerguelen but also at Crozet and Heard islands (Weimerskirch et al. 1986, Stahl et al. in press). Elsewhere, breeding black-browed albatrosses are apparently restricted to the shelf (Falklands: Thompson 1992), or spend most time over neritic waters, foraging also in the oceanic zone (South Georgia: Prince et al. 1998, Campbell Island: Waugh et al. 1999, in press). Furthermore, birds from different populations, including the Kerguelen albatrosses, winter over the South America, Patagonian, South Africa and Australian shelves (Prince et al. 1998, Cherel et al. 2000), thus leading to the conclusion that the black-browed albatross is essentially a neritic-slope predator. Ecologically, the species belongs to a group of albatrosses that use primarily shelf and shelf-break waters, including the shy albatross Diomedea cauta in southern Australia (Brothers et al. 1998), and Buller's albatross D. bulleri (Stahl et al. 1998) and royal albatross D. epomophora (Imber 1999) in New Zealand.

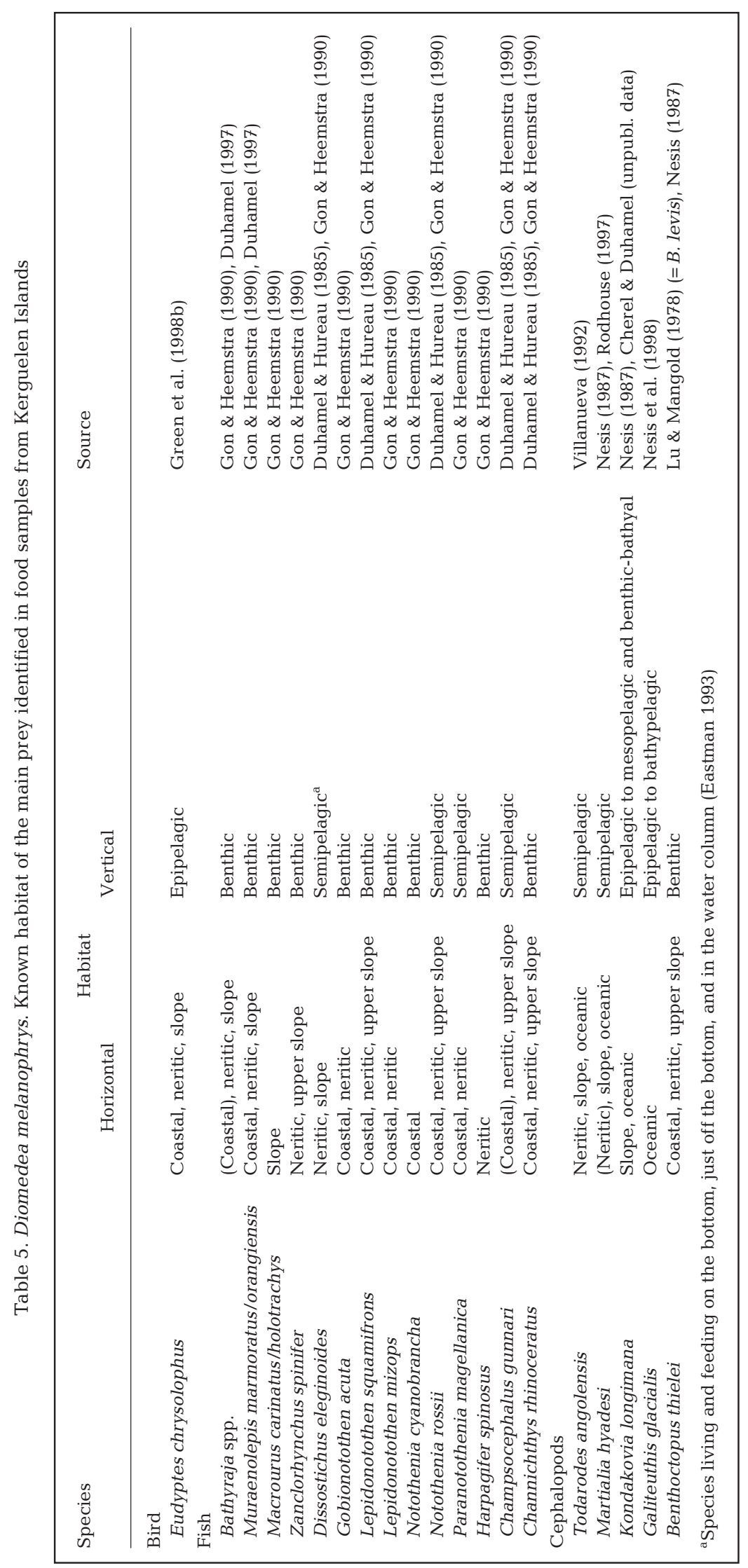




\section{Benthic fish and cephalopods: natural prey or fishery waste?}

At Kerguelen, albatrosses preyed upon some epipelagic organisms, including penguins and the squids Martialia hyadesi and Todarodes sp. These ommastrephids extend from the epipelagic to the bottom. Juveniles are associated with the upper layers of the water column (Villanueva 1992, Rodhouse \& White 1995), and sometimes with the surface where blackbrowed albatrosses had been observed feeding on them (Nolan et al. 1998). The food of albatrosses at Kerguelen is consistant with this mode of life since almost all the ommastrephids were juveniles (Fig. 4). The lack of young $M$. hyadesi and Todarodes sp. in samples collected in late March/early April suggests also that the ontogenic migration to the bottom occurred at that time, squids becoming thus unavailable to the birds.

Most of the albatross prey at Kerguelen are considered to be benthic or semipelagic organisms (Table 5). This is particularly obvious for fish, because epipelagic fish families are absent from both the Antarctic Polar Frontal Zone and the Antarctic Zone (Rodhouse \& White 1995). Despite this, fish, mainly of the families Nototheniidae and Channichthyidae, are a major component of the food of albatrosses in the Southern Ocean (Cherel \& Klages 1998, this study). The question arises, how do flying seabirds regularly capture organisms of essentially benthic habitat? The commonest explanation refers to links with fishery operations. It is assumed that the birds feed on fishery waste, including offal and discarded items (Reid et al. 1996, Imber 1999). At Kerguelen, black-browed albatrosses indeed attended trawlers and longliners in large numbers during the study period (Weimerskirch et al. 2000). A close examination of dietary samples indicate that some remains from Dissostichus eleginoides were probably offal. These items consisted of pieces of vertebrae and tails from individual fish far too large to be swallowed whole by albatrosses. A likely explanation is that the birds from Canyon des Sourcils Noirs attended the French trawler targetting the $D$. eleginoides in the north of the archipelago, since this vessel provided a large quantity of offal in a zone that is a natural foraging area for albatrosses (Figs. $1 \& 5$ ). Offal and/or hooks had been recently found in stomach contents of whitechinned petrels and wandering albatrosses (Catard et al. 2000, authors' unpubl. data). The birds ate fish and squid known to be used as bait by longliners. The lack of bait remains and hooks, together with the location of the Ukrainian longline fishery targetting the $D$. eleginoides on the western shelf, indicates little interaction between the fishery and black-browed albatrosses from Canyon des Sourcils Noirs. Adult birds however consistently attend Ukrainian longliners (Cherel et al. 1996, Weimerskirch et al. 2000) in an area where birds from the Nuageuses Islands forage (Weimerskirch et al. 1988). This suggests that breeding individuals from that colony are more at risk of being killed on hooks.

Unlike offal, the amount of fishery discards was quite limited with a maximum of 7 fishing vessels in the area, the 3 longliners taking skates too large to be swallowed by albatrosses (Cherel et al. 1996), and the 4 trawlers discarding a few fish and squid (Weimerskirch et al. 2000). Note that, to our knowledge, no poaching trawlers and longliners occurred in the area in 1994 and 1995, thus precluding a larger availability of fishery wastes for albatrosses (see 'Methods'). Seabirds can also feed during hauling operations when the net is at some distance from the boat. The occurrence of Ukrainian trawlers targetting Champsocephalus gunnari in 1995 was associated with a weak increase in the proportion of C. gunnari in the albatross food at that time when compared to 1994 (2.6 vs $7.0 \%$ in 1994 and 1995, respectively; $\left.\chi^{2}{ }_{1}=2.98, p=0.085\right)$. This suggests that albatrosses did not rely heavily on discarded fish and squids and on the targetted species, the only important fishery waste being offal from Dissostichus eleginoides. Albatrosses moreover are able to feed by themselves on large $D$. eleginoides, as this species was the main fish prey of wandering albatrosses Diomedea exulans at the Crozet Islands during the austral winter of 1992 (Weimerskirch et al. 1997a), when no fishery occurred in the area.

Comparison between albatross foraging zones and fishery areas showed overlap with the trawl fisheries north of the archipelago both in February 1994 and 1995. Temporal analysis between and within years, however, indicates that albatrosses foraged there even in the absence of fishing vessels. This suggests that the northern shelf is a natural feeding zone for seabirds. At Kerguelen (Duhamel 1981, this study), as elsewhere, spatial overlap between fisheries and predator foraging grounds is not unexpected since both favor productive zones where high prey biomass concentrates. Most of the satellite fixes from albatrosses were located outside the 1994-1995 commercial fishing grounds. For example, no vessels worked east of the mainland, which was the most important foraging area for black-browed albatrosses. Prey associated with known trips also showed that albatrosses were able to feed by themselves on demersal prey like skates, Channichthys rhinoceratus and Benthoctopus thielei. A major finding of this work therefore is that it seems unlikely that the birds acquired most of the benthic and semipelagic organisms through the commercial fisheries. They were thus natural prey of black-browed albatrosses at Kerguelen Islands. This is probably true elsewhere because nototheniids and channichthyids 
are the main fish prey of albatrosses from the Southern Ocean (Cherel \& Klages 1998). Therefore, care is needed when evaluating trophic interactions between seabirds and fisheries by only measuring the importance of benthic and semipelagic prey in their diet.

How benthic organisms become available for predators foraging at the surface and in the top few meters of the water column (Prince et al. 1994, Hedd et al. 1997, Huin \& Prince 1997) is therefore unclear. Notothenia rossii has been observed feeding near the surface (Shust \& Sil'yanova 1971, cited in Duhamel \& Hureau 1985), and some benthic organisms, including skates, Zanclorhynchus spinifer, Channichthys rhinoceratus and Benthoctopus thielei were found dead or dying on the shoreline (Clarke 1850, Arnaud 1972, authors' unpubl. data). This suggests, unlike stated by Reid et al. (1996), that black-browed albatrosses obtained a substantial amount of food by scavenging dead floating cephalopods and fish. Postspawning individuals found at the surface is the more likely explanation of birds feeding on the adult squids Galiteuthis glacialis and Kondakovia longimana (Nesis et al. 1998, Cherel \& Weimerskirch 1999). This may also be true for fish (Croxall et al. 1988). However, most of the fish eaten by albatrosses had not yet reached adult size, except Notothenia cyanobrancha and some channichthyids (Gon \& Heemstra 1990, Table 4).

Seabirds are known to feed on prey forced to the surface by sub-surface foragers like marine mammals and tuna (Ballance \& Pitman 1999). Such a feeding behaviour was described for black-browed albatrosses preying on Euphausia superba driven to the surface by fur seals and penguins at South Georgia (Harrison et al. 1991). The species has also been observed feeding in association with killer whales at the Crozet Islands (Ridoux 1987), but this was not recorded in Kerguelen waters (authors' unpubl. data). Clearly, more information is needed, not only on the feeding ecology of apex predators, but also on the biology of their prey to understand the trophic relationships between large flying seabirds and the benthic resources of the Southern Ocean.

Acknowledgements. The authors thank Guy Duhamel and French fishery observers for providing us data on commercial fisheries, and intact fish and cephalopods from Kerguelen waters, and Dominique Beauvais, Pierrick Bochet, Bruno Labidoire and Thierry Mougey for their help in the field. Analysis of the data was supported financially by a grant from the Groupement de Recherche en Environnement 1069 'Ecosystèmes Polaires et Anthropisation' (director Pierre Jouventin), from the CNRS Field work was supported financially and logistically by the Institut Français pour la Recherche et la Technologie Polaires (IFRTP, Programme No. 109), and the Terres Australes et Antarctiques Françaises.

\section{LITERATURE CITED}

Adams NJ, Klages NT (1987) Seasonal variation in the diet of the king penguin (Aptenodytes patagonicus) at subAntarctic Marion Island. J Zool Lond 212:303-324

Andersen NC (1984) Genera and subfamilies of the family Nototheniidae (Pisces, Perciformes) from the Antarctic and Subantarctic. Steenstrupia 10:1-34

Arnaud PM (1972) Notes écologiques sur Zanclorhynchus spinifer (Téléostéen, Congiopodidae) aux Iles Kerguelen et sur sa prédation par les pétrels géants Macronectes spp. Tethys 4:757-760

Baker Ade C, Boden BP, Brinton E (1990) A practical guide to the euphausiids of the world. Nat His Mus Publ, London

Ballance LT, Pitman RL (1999) Foraging ecology of tropical seabirds. In: Adams NJ, Slotow RH (eds) Proc 22nd Int Ornithol Congr Durban. BirdLife South Africa, Durban, p 2057-2071

Blaber SJM, Milton DA, Smith GC, Farmer MJ (1995) Trawl discards in the diets of tropical seabirds of the northern Great Barrier Reef, Australia. Mar Ecol Prog Ser 127:1-13

Branch ML, Griffiths CL, Kensley B, Sieg J (1991) The benthic Crustacea of subantarctic Marion and Prince Edward Islands: illustrated keys to the species and results of the 1982-1989 University of Cape Town Surveys. S Afr J Antarct Res 21:3-44

Brothers N, Gales R, Hedd A, Robertson G (1998) Foraging movements of the shy albatross Diomedea cauta breeding in Australia; implications for interactions with longline fisheries. Ibis 140:446-457

Cairns DK (1992) Population regulation of seabird colonies. Current Ornithol 9:37-61

Catard A, Weimerskirch H, Cherel Y (2000) Exploitation of distant Antarctic waters and close shelf-break waters by white-chinned petrels rearing chicks. Mar Ecol Prog Ser 194:249-261

Cherel Y, Klages N (1998) A review of the food of albatrosses. In: Robertson G, Gales R (eds) Albatross biology and conservation. Surrey Beatty and Sons, Chipping Norton, p 113-136

Cherel Y, Weimerskirch H (1995) Seabirds as indicators of marine resources: black-browed albatrosses feeding on ommastrephid squids in Kerguelen waters. Mar Ecol Prog Ser 129:295-300

Cherel Y, Weimerskirch H (1999) Spawning cycle of onychoteuthid squids in the southern Indian Ocean: new information from seabird predators. Mar Ecol Prog Ser 188: 93-104

Cherel Y, Charrassin JB, Challet E (1994) Energy and protein requirements for molt in the king penguin Aptenodytes patagonicus. Am J Physiol 266:R1182-R1188

Cherel Y, Weimerskirch H, Duhamel G (1996) Interactions between longline vessels and seabirds in Kerguelen waters and a method to reduce seabird mortality. Biol Conserv 75:63-70

Cherel Y, Waugh S, Hanchet S (1999) Albatross predation of juvenile southern blue whiting (Micromesistius australis) on the Campbell Plateau. NZ J Mar Freshw Res 33: 437-441

Cherel Y, Hobson KA, Weimerskirch H (2000) Using stableisotope analysis of feathers to distinguish moulting and breeding origins of seabirds. Oecologia 122:155-162

Clarke A, Holmes LJ (1987) Notes on the biology and distribution of Pasiphaea species from the Southern Ocean. Br Antarct Surv Bull 74:17-30

Clarke MR (1986) A handbook for the identification of cephalopod beaks. Clarendon Press, Oxford 
Clarke WB (1850) Récit du naufrage de la Favorite aux îles de la Désolation relatant les aventures, souffrances et privations de John Nunn. In: Nougier J (ed) Trois naufrages pour trois îles, Terres Australes Françaises au XIX ${ }^{\mathrm{e}}$ siècle. Editions de la Dyle, Aix-en-Provence, p 83-228, 1998

Cooper J, Klages NTW (1995) The diets and dietary segregation of sooty albatrosses (Phoebetria spp.) at subantarctic Marion Island. Antarct Sci 7:15-23

Cooper J, Henley SR, Klages NTW (1992) The diet of the wandering albatross Diomedea exulans at Subantarctic Marion Island. Polar Biol 12:477-484

Croxall JP, North AW, Prince PA (1988) Fish prey of the wandering albatross Diomedea exulans at South Georgia. Polar Biol 9:9-16

Croxall JP, Hall AJ, Hill HJ, North AW, Rodhouse PG (1995) The food and feeding ecology of the white-chinned petrel Procellaria aequinoctialis at South Georgia. J Zool Lond 237:133-150

DeGange AR, Day RH (1991) Mortality of seabirds in the Japanese land-based gillnet fishery for salmon. Condor 93:251-258

Duffy DC, Jackson S (1986) Diet studies of seabirds: a review of methods. Colon Waterbirds 9:1-17

Duhamel G (1981) Caractéristiques biologiques des principales espèces de poissons du plateau continental des îles Kerguelen. Cybium 5:19-32

Duhamel G (1997) L'ichtyofaune des îles australes françaises de l'Océan Indien. Cybium 21(Suppl):147-168

Duhamel G (1998) The pelagic fish community of the Polar Frontal Zone off the Kerguelen Islands. In: di Prisco G, Pisano E, Clarke A (eds) Fishes of Antarctica. A biological overview. Springer Verlag, Berlin, p 63-74

Duhamel G, Hureau JC (1985) The role of zooplankton in the diets of certain sub-Antarctic marine fish. In: Siegfried WR, Condy PR, Laws RM (eds) Antarctic nutrient cycles and food webs. Springer Verlag, Berlin, p 421-429

Duhamel G, Pruvost P, Capdeville D (1997) By-catch of fish in longline catches off the Kerguelen Islands (Division 58.5.1) during the 1995/96 season. CCAMLR Sci 4: 175-193

Eastman JT (1993) Antarctic fish biology. Evolution in a unique environment. Academic Press, San Diego

Freeman AND (1998) Diet of Westland petrels Procellaria westlandica: the importance of fisheries waste during chick-rearing. Emu 98:36-43

Garthe S, Camphuysen KCJ, Furness RW (1996) Amounts of discards by commercial fisheries and their significance as food for seabirds in the North Sea. Mar Ecol Prog Ser 136: $1-11$

Gon O, Heemstra PC (1990) Fishes of the Southern Ocean. JLB Smith Institute of Ichthyology, Grahamstown

Gosztonyi AE, Kuba L (1998) Fishes in the diet of the imperial cormorant Phalacrocorax atriceps at Punta Loberia Chubut, Argentina. Mar Ornithol 26:59-61

Green K, Kerry KR, Disney T, Clarke MR (1998a) Dietary studies of light-mantled sooty albatrosses Phoebetria palpebrata from Macquarie and Heard Islands. Mar Ornithol 26:19-26

Green K, Williams R, Green MG (1998b) Foraging ecology and diving behaviour of macaroni penguins Eudyptes chrysolophus at Heard Island. Mar Ornithol 26:27-34

Guerra A, Gonzalez AF, Cherel Y (2000) Graneledone gonzalezi sp. nov. (Mollusca: Cephalopoda): a new octopod from the Iles Kerguelen. Antarct Sci 12:33-40

Harrison CS, Hida TS, Seki MP (1983) Hawaiian seabird feeding ecology. Wildl Monogr 85:1-71

Harrison NM, Whitehouse MJ, Heinemann D, Prince PA,
Hunt GL, Veit RR (1991) Observations of multispecies seabird flocks around South Georgia. Auk 108:801-810

Hedd A, Gales R, Brothers N, Robertson G (1997) Diving behaviour of the shy albatross Diomedea cauta in Tasmania: initial findings and dive recorder assessment. Ibis 139: $452-460$

Huin N, Prince PA (1997) Diving behaviour of the greyheaded albatross. Antarct Sci 9:243-249

Hunt GL, Mehlum F, Russell RW, Irons D, Decker MB, Becker PH (1999) Physical processes, prey abundance, and the foraging ecology of seabirds. In: Adams NJ, Slotow RH (eds) Proc 22nd Int Ornithol Congr Durban. BirdLife South Africa, Durban, p 2040-2056

Hunter S, NTW Klages (1989) The diet of grey-headed albatrosses Diomedea chrysostoma at the Prince Edward Islands. S Afr J Antarct Res 19:31-33

Hureau JC (1970) Biologie comparée de quelques poissons antarctiques (Nototheniidae). Bull Inst Océanogr Monaco 68:1-244

Imber MJ (1999) Diet and feeding ecology of the royal albatross Diomedea epomophora - king of the shelf-break and inner slope. Emu 99:200-211

Iwami T (1985) Osteology and relationships of the family Channichthyidae. Mem Nat Inst Polar Res E 36:1-69

Jackson GD (1995) The use of beaks as tools for biomass estimation in the deepwater squid Moroteuthis ingens (Cephalopoda: Onychoteuthidae) in New Zealand waters. Polar Biol 15:9-14

Lu CC, Mangold K (1978) Cephalopods of the Kerguelen Province of the Indian Ocean. In: Proceedings of the International Symposium on marine biogeography and evolution in the Southern Hemisphere, Auckland, Vol. 2, p 567-574

Marchant S, Higgins PJ (1990) Handbook of Australian, New Zealand and Antarctic birds, Vol 1. Oxford University Press, Melbourne

Montevecchi WA (1993) Birds as indicators of change in marine prey stocks. In: Furness RW, Greenwood JJD (eds) Birds as monitors of environmental changes. Chapman \& Hall, London, p 217-266

Murano M (1999) Mysidacea. In: Boltovskoy D (ed) South Atlantic zooplankton. Backhuys Publishers, Leiden, p 1099-1140

Nesis KN (1987) Cephalopods of the world. Squids, cuttlefishes, octopuses and allies. TFH Publications, Neptune City, NJ

Nesis KN, Nigmatullin CM, Nikitina IV (1998) Spent females of deepwater squid Galiteuthis glacialis under the ice at the surface of the Weddell Sea (Antarctic). J Zool Lond 244:185-200

Nolan CP, Strange IJ, Alesworth E, Agnew DJ (1998) A mass stranding of the squid Martialia hyadesi Rochebrune and Mabille, 1889 (Teuthoidea: Ommastrephidae) at New Island, Falkland Islands. S Afr J Mar Sci 20:305-310

Prince PA (1980) The food and feeding ecology of greyheaded albatross Diomedea chrysostoma and blackbrowed albatross D. melanophris. Ibis 122:476-488

Prince PA, Huin N, Weimerskirch H (1994) Diving depths of albatrosses. Antarct Sci 6:353-354

Prince PA, Croxall JP, Trathan PN, Wood AG (1998) The pelagic distribution of South Georgia albatrosses and their relationships with fisheries. In: Robertson G, Gales R (eds) Albatross biology and conservation. Surrey Beatty and Sons, Chipping Norton, p 137-167

Reid K, Croxall JP, Prince PA (1996) The fish diet of blackbrowed albatross Diomedea melanophris and greyheaded albatross D. chrysostoma at South Georgia. Polar Biol 16:469-477 
Ridoux V (1987) Feeding association between seabirds and killer whales, Orcinus orca, around subantarctic Crozet Islands. Can J Zool 65:2113-2115

Ridoux V (1994) The diets and dietary segregation of seabirds at the subantarctic Crozet Islands. Mar Ornithol 22:1-192

Robertson G, Gales R (1998) Albatross biology and conservation. Surrey Beatty \& Sons, Chipping Norton

Rodhouse PG (1997) Large and meso-scale distribution of the ommastrephid squid Martialia hyadesi in the Southern Ocean: a synthesis of information relevant to fishery forecasting and management. Korean J Polar Res 8:145-154

Rodhouse PG, Prince PA (1993) Cephalopod prey of the black-browed albatross Diomedea melanophrys at South Georgia. Polar Biol 13:373-376

Rodhouse PG, White MG (1995) Cephalopods occupy the ecological niche of epipelagic fish in the Antarctic Polar Frontal Zone. Biol Bull 189:77-80

Rodhouse PG, Yeatman J (1990) Redescription of Martialia hyadesi Rochebrune and Mabille, 1889 (Mollusca: Cephalopoda) from the Southern ocean. Bull Br Mus Nat Hist (Zool) 56:135-143

Stahl JC, Bartle JA, Cheshire NG, Petyt C, Sagar PM (1998) Distribution and movements of Buller's albatross (Diomedea bulleri) in Australasian seas. NZ J Zool 25:109-137

Stahl JC, Bartle JA, Jouventin P, Roux JP, Weimerskirch H (in press) Atlas of seabird distribution in the South-west Indian Ocean. Mar Ornithol

Thomas G (1982) The food and feeding ecology of the lightmantled sooty albatross at South Georgia. Emu 82:92-100

Thompson KR (1992) Quantitative analysis of the use of discards from squid trawlers by black-browed albatrosses Diomedea melanophris in the vicinity of the Falkland Islands. Ibis 134:11-21

Villanueva R (1992) Interannual growth differences in the oceanic squid Todarodes angolensis Adam in the northern Benguela upwelling system, based on statolith growth increment analysis. J Exp Mar Biol Ecol 159:157-177

Vinogradov ME, Volkov AF, Semenova TN (1996) Hyperiid

Editorial responsibility: Otto Kinne (Editor), Oldendorf/Luhe, Germany amphipods (Amphipoda, Hyperiidea) of the world oceans. Science Publishers, Lebanon

Waugh SM, Weimerskirch H, Cherel Y, Shankar U, Prince PA, Sagar PM (1999) Exploitation of the marine environment by two sympatric albatrosses in the Pacific Southern Ocean. Mar Ecol Prog Ser 177:243-254

Waugh SM, Weimerskirch H, Cherel Y, Prince PA (in press) Contrasting strategies of provisioning and chick growth in two sympatrically breeding albatrosses at Campbell Island, New Zealand. Condor

Weimerskirch H, Jouventin P, Stahl JC (1986) Comparative ecology of the six albatross species breeding on the Crozet Islands. Ibis 128:195-213

Weimerskirch H, Bartle JA, Jouventin P, Stahl JC (1988) Foraging ranges and partitioning of feeding zones in three species of southern albatrosses. Condor 90:214-219

Weimerskirch H, Zotier R, Jouventin P (1989) The avifauna of the Kerguelen Islands. Emu 89:15-29

Weimerskirch H, Salamolard M, Sarrazin F, Jouventin P (1993) Foraging strategy of wandering albatrosses through the breeding season: a study using satellite telemetry. Auk 110:325-342

Weimerskirch $\mathrm{H}$, Cherel $\mathrm{Y}$, Cuénot-Chaillet $\mathrm{F}$, Ridoux V (1997a) Alternative foraging strategies and resource allocation by male and female wandering albatrosses. Ecology 78:2051-2063

Weimerskirch H, Mougey T, Hindermeyer X (1997b) Foraging and provisioning strategies of black-browed albatrosses in relation to the chick: natural variation and experimental study. Behav Ecol 8:635-643

Weimerskirch H, Capdeville D, Duhamel G (2000) Factors affecting the number and mortality of seabirds attending trawlers and long-liners in the Kerguelen area. Polar Biol 23:236-249

Wilkinson L (1997) SYSTAT 7.0 for Windows. SPSS, Chicago

Williams R, McEldowney A (1990) A guide to the fish otoliths from waters off the Australian Antarctic Territory, Heard and Macquarie Islands. ANARE Res Notes 75:1-173

Submitted: February 28, 2000; Accepted: May 25, 2000 Proofs received from author(s): October 27, 2000 